TRANSACTIONS OF THE

AMERICAN MATHEMATICAL SOCIETY

Volume 361, Number 6, June 2009, Pages 3303-3323

S 0002-9947(09)04805-3

Article electronically published on January 28, 2009

\title{
GREEN'S MATRICES OF SECOND ORDER ELLIPTIC SYSTEMS WITH MEASURABLE COEFFICIENTS IN TWO DIMENSIONAL DOMAINS
}

\author{
HONGJIE DONG AND SEICK KIM
}

\begin{abstract}
We study Green's matrices for divergence form, second order strongly elliptic systems with bounded measurable coefficients in two dimensional domains. We establish existence, uniqueness, and pointwise estimates of Green's matrices.
\end{abstract}

\section{INTRODUCTION}

In this article, we study Green's matrices for divergence form, second order strongly elliptic systems with bounded measurable coefficients in two dimensional domains. More precisely, we are concerned with the Green's matrix for elliptic systems

$$
\sum_{j=1}^{N} L_{i j} u^{j}:=\sum_{j=1}^{N} \sum_{\alpha, \beta=1}^{2} D_{\alpha}\left(A_{i j}^{\alpha \beta}(x) D_{\beta} u^{j}\right), \quad i=1, \ldots, N,
$$

in an open connected set $\Omega \subset \mathbb{R}^{2}$. Here, $A_{i j}^{\alpha \beta}(x)$ are bounded measurable functions on $\Omega$ satisfying the strong ellipticity condition.

By a Green's matrix we mean an $N \times N$ matrix valued function $\boldsymbol{G}(x, y)=$ $\left(G_{i j}(x, y)\right)_{i, j=1}^{N}$ defined on $\{(x, y) \in \Omega \times \Omega: x \neq y\}$ satisfying the following properties (see Theorem 2.12 below for a more precise statement):

$$
\begin{aligned}
& \sum_{j=1}^{N} L_{i j} G_{j k}(\cdot, y)=-\delta_{i k} \delta_{y}(\cdot) \quad \forall y \in \Omega, \\
& G_{i j}(\cdot, y)=0 \quad \text { on } \quad \partial \Omega \quad \forall y \in \Omega,
\end{aligned}
$$

where $\delta_{i k}$ is the Kronecker delta symbol and $\delta_{y}(\cdot)$ is the Dirac delta function with a unit mass at $y$. In the scalar case (i.e., when $N=1$ ), the Green's matrix becomes a real valued function and is usually called the Green's function.

Received by the editors September 5, 2007.

2000 Mathematics Subject Classification. Primary 35A08, 35B65; Secondary 35J45.

Key words and phrases. Green function, Green's matrix, fundamental solution, fundamental matrix, second order elliptic system, measurable coefficients.

The first author was partially supported by the National Science Foundation under agreement No. DMS-0111298 and a start-up funding from the Division of Applied Mathematics of Brown University.

The second author was supported by the Australian Research Council and by the New Faculty Research Grant No. 2008-1-0010 from Yonsei University.

(C)2009 American Mathematical Society Reverts to public domain 28 years from publication 
We prove that if $\Omega$ has either finite volume or finite width, then there exists a unique Green's matrix in $\Omega$; see Theorem 2.12 The same is true when $\Omega$ is a domain above a Lipschitz graph (e.g., $\Omega=\mathbb{R}_{+}^{2}$ ); see Theorem 2.21 . We also establish growth properties of the Green's matrices including logarithmic pointwise bounds. We emphasize that we do not require $\Omega$ to be bounded nor to have a regular boundary in Theorem 2.12. Compared to the result of Dolzmann and Müller [4, where $\Omega$ is assumed to be a bounded Lipschitz domain (in fact, their methods work whenever there exists an $L^{p}$-theory for the equation under consideration on the domain), our result is quite an improvement in this respect. Although there is no Green's matrix for $\Omega=\mathbb{R}^{2}$, there is a possible definition of a fundamental matrix in the entire plane. Such a construction was carried out by Kenig and Ni 12] in the scalar case and by Auscher, McIntosh, and Tchamitchian [1] in the systems setting. (In fact, Auscher et al. considered elliptic equations with complex coefficients in [1], but with appropriate changes their strategy carries over to more general elliptic systems.) For the completeness of presentation, we include the result of Auscher et al. [1] in Section 5 .

Let us briefly review the history of works in this area. In the scalar case, the basic facts about Green's functions of symmetric elliptic operators in bounded domains were proved by Littman, Stampacchia, and Weinberger [16. The study of the Green's functions for nonsymmetric elliptic operators in bounded domains $\Omega \subset \mathbb{R}^{n}$ $(n \geq 3)$ was carried out by Grüter and Widman $[9$. As it was mentioned earlier, there is no Green's function for $\Omega=\mathbb{R}^{2}$; the fundamental solution $-(1 / 2 \pi) \ln |x-y|$ of the Laplace equation changes sign and is not considered a Green's function from a point of view of classical potential theory (see, e.g., [5]). Nevertheless, it is still possible to define a fundamental solution in $\mathbb{R}^{2}$. By using the maximum principle, Kenig and $\mathrm{Ni}$ [12] constructed one for symmetric elliptic operators. In 2], Chanillo and $\mathrm{Li}$ derived that the fundamental solution constructed by Kenig and $\mathrm{Ni}$ is a function of bounded mean oscillation in $\mathbb{R}^{2}$. Also, we would like to bring the reader's attention to a paper by Escauriaza [6] on the fundamental solutions of elliptic and parabolic equations in nondivergence form. In the systems setting, the Green's matrices of the elliptic systems with continuous coefficients in bounded $C^{1}$ domains have been discussed by Fuchs 7 and Dolzmann and Müller [4]. In fact, Dolzmann and Müller improved the strategy of Fuchs and showed the existence and pointwise estimate for Green's matrix in bounded Lipschitz domains $\Omega \subset \mathbb{R}^{2}$ without imposing any regularity on the coefficients (their methods work whenever an $L^{p}$ theory is available for the domain $\Omega$ ). Recently, Hofmann and Kim [10] gave a unified approach in studying Green's functions/matrices in arbitrary domains $\Omega \subset \mathbb{R}^{n}(n \geq 3)$ valid for both scalar equations and systems of elliptic type by considering a class of operators $L$ such that weak solutions of $L \boldsymbol{u}=0$ satisfy an interior Hölder estimate. However, like the method used in Grüter and Widman [9], the method of Hofmann and Kim relied heavily on the assumption that $n \geq 3$ and could not be applied to the two dimensional case. A parabolic extension of the result by Hofmann and Kim was carried out in a very recent paper by Cho, Dong, and Kim 3. In particular, Cho et al. proved that the so-called "Dirichlet heat kernel" of a strongly elliptic system exists in any domain $\Omega \subset \mathbb{R}^{2}$ (see Corollary 2.9 in [3). In fact, our basic strategy is to make use of their result and construct the Green's matrix out of the "Dirichlet heat kernel" by integrating in a $t$-variable.

The organization of this paper is as follows. In Section 2, we introduce some notation and then state our main results, Theorem 2.12 and Theorem 2.21. We 
give the proof of Theorem 2.12 in Section 3 and that of Theorem 2.21 in Section 4 Finally, in Section 5 we introduce the result of Auscher et al. [1] regarding construction of a fundamental matrix for an elliptic system in the entire plane.

\section{Preliminaries AND MAin Results}

2.1. Strongly elliptic systems in $\mathbb{R}^{2}$. Throughout this article, the summation convention over repeated indices shall be assumed. Let $L$ be a second order elliptic operator of divergence type acting on vector valued functions $\boldsymbol{u}=\left(u^{1}, \ldots, u^{N}\right)^{T}$ $(N \geq 1)$ defined on an open set $\Omega \subset \mathbb{R}^{2}$ in the following way:

$$
L \boldsymbol{u}=D_{\alpha}\left(\boldsymbol{A}^{\alpha \beta} D_{\beta} \boldsymbol{u}\right) \quad\left(:=\sum_{\alpha=1}^{2} \sum_{\beta=1}^{2} D_{\alpha}\left(\boldsymbol{A}^{\alpha \beta} D_{\beta} \boldsymbol{u}\right)\right),
$$

where $\boldsymbol{A}^{\alpha \beta}=\boldsymbol{A}^{\alpha \beta}(x)(\alpha, \beta=1,2)$ are $N$ by $N$ matrices satisfying the strong ellipticity condition; i.e., there is a number $\lambda>0$ such that

$$
A_{i j}^{\alpha \beta}(x) \xi_{\beta}^{j} \xi_{\alpha}^{i} \geq \lambda|\boldsymbol{\xi}|^{2}:=\lambda \sum_{i=1}^{N} \sum_{\alpha=1}^{2}\left|\xi_{\alpha}^{i}\right|^{2} \quad \forall x \in \Omega .
$$

We also assume that $A_{i j}^{\alpha \beta}$ are bounded; i.e., there is a number $\Lambda>0$ such that

$$
\sum_{i, j=1}^{N} \sum_{\alpha, \beta=1}^{2}\left|A_{i j}^{\alpha \beta}(x)\right|^{2} \leq \Lambda^{2} \quad \forall x \in \Omega .
$$

We do not impose any further condition other than (2.2) and (2.3) on the coefficients. Especially, we do not assume the symmetry of the coefficients. The transpose operator ${ }^{t} L$ of $L$ is defined by

$$
{ }^{t} L \boldsymbol{u}=D_{\alpha}\left({ }^{t} \boldsymbol{A}^{\alpha \beta} D_{\beta} \boldsymbol{u}\right),
$$

where ${ }^{t} \boldsymbol{A}^{\alpha \beta}=\left(\boldsymbol{A}^{\beta \alpha}\right)^{T}$ (i.e., ${ }^{t} A_{i j}^{\alpha \beta}=A_{j i}^{\beta \alpha}$ ). Note that the coefficients ${ }^{t} A_{i j}^{\alpha \beta}$ satisfy the conditions (2.2), (2.3) with the same constants $\lambda, \Lambda$.

2.2. The function space $Y_{0}^{1,2}(\Omega)$. The function space $Y_{0}^{1,2}(\Omega)$ is defined as the set of all weakly differentiable functions on $\Omega$ such that $D u \in L^{2}(\Omega)$ and $u \eta \in W_{0}^{1,2}(\Omega)$ for any $\eta \in C_{c}^{\infty}\left(\mathbb{R}^{2}\right)$. An open set $\Omega \subset \mathbb{R}^{2}$ is said to be a Green domain if $\left\{u 1_{\Omega}: u \in C_{c}^{\infty}\left(\mathbb{R}^{2}\right)\right\} \not \subset W_{0}^{1,2}(\Omega)$. We ask the readers to refer to [17, 11.3 .4$]$ for the proofs of lemmas stated below.

Lemma 2.5. Let $\Omega \subset \mathbb{R}^{2}$ be a Green domain and $B \subset \mathbb{R}^{2}$ be a ball. Then, there is a constant $C=C(\Omega, B)$ such that

$$
\|u\|_{L^{2}(\Omega \cap B)} \leq C\|D u\|_{L^{2}(\Omega)} \quad \forall u \in Y_{0}^{1,2}(\Omega) .
$$

Lemma 2.7. Let $\Omega \subset \mathbb{R}^{2}$ be a Green domain. Then $Y_{0}^{1,2}(\Omega)$ is a Hilbert space when endowed with the inner product

$$
\langle u, v\rangle:=\int_{\Omega} D_{i} u D_{i} v
$$


Lemma 2.9. Let $\Omega \subset \mathbb{R}^{2}$ be a Green domain. Then $C_{c}^{\infty}(\Omega)$ is a dense subset of the Hilbert space $Y_{0}^{1,2}(\Omega)$ equipped with the inner product (2.8).

For a given function $\boldsymbol{f}=\left(f^{1}, \ldots, f^{N}\right)^{T} \in L_{l o c}^{1}(\Omega)^{N}$, we shall say that $\boldsymbol{u}=$ $\left(u^{1}, \ldots, u^{N}\right)^{T}$ is a weak solution in $Y_{0}^{1,2}(\Omega)^{N}$ of $L \boldsymbol{u}=-\boldsymbol{f}$ if $\boldsymbol{u} \in Y_{0}^{1,2}(\Omega)^{N}$ and

$$
\int_{\Omega} A_{i j}^{\alpha \beta} D_{\beta} u^{j} D_{\alpha} \phi^{i}=\int_{\Omega} f^{i} \phi^{i} \quad \forall \phi \in C_{c}^{\infty}(\Omega)^{N} .
$$

It is routine to check that if $\Omega$ is a Green domain and $\boldsymbol{u}$ is a weak solution in $Y_{0}^{1,2}(\Omega)^{N}$ of $L \boldsymbol{u}=0$, then $\boldsymbol{u} \equiv 0$. Therefore, a weak solution in $Y_{0}^{1,2}(\Omega)^{N}$ of $L \boldsymbol{u}=-\boldsymbol{f}$ is unique.

2.3. Main results. Let us state our main results. First, we consider domains with either finite volume or finite width. We shall denote by $|\Omega|$ the Lebesgue measure of $\Omega$ and by $\delta(\Omega)$ the width of $\Omega \subset \mathbb{R}^{2}$; more precisely, we define

$$
\delta(\Omega):=\inf \left\{\operatorname{dist}\left(\ell_{1}, \ell_{2}\right): \Omega \text { lies between two parallel lines } \ell_{1}, \ell_{2}\right\} ; \quad \inf \emptyset=\infty \text {. }
$$

Theorem 2.12. Let the operator $L$ satisfy the conditions (2.2) and (2.3). Assume that $\Omega \subset \mathbb{R}^{2}$ is an open connected set with either finite volume or finite width so that

$$
\gamma=\gamma(\Omega):=\max \left(|\Omega|^{-1}, \delta(\Omega)^{-2}\right)>0 .
$$

Then, there exists a Green's matrix $\boldsymbol{G}(x, y)=\left(G_{i j}(x, y)\right)_{i, j=1}^{N}$ defined on $\{(x, y) \in$ $\Omega \times \Omega: x \neq y\}$ satisfying the properties that

$$
\int_{\Omega} A_{i j}^{\alpha \beta} D_{\beta} G_{j k}(\cdot, y) D_{\alpha} \phi^{i}=\phi^{k}(y) \quad \forall \phi \in C_{c}^{\infty}(\Omega)^{N}
$$

and that for all $\eta \in C_{c}^{\infty}(\Omega)$ satisfying $\eta \equiv 1$ on $B_{r}(y)$ for some $r<d_{y}$,

$$
(1-\eta) \boldsymbol{G}(\cdot, y) \in Y_{0}^{1,2}(\Omega)^{N \times N} .
$$

The Green's matrix $\boldsymbol{G}(x, y)$ in $\Omega$ is unique in the following sense:

(a) $\boldsymbol{G}(x, y)$ is continuous in $\{(x, y) \in \Omega \times \Omega: x \neq y\}$.

(b) $\boldsymbol{G}(x, \cdot)$ is locally integrable for all $x \in \Omega$.

(c) For any $\boldsymbol{f}=\left(f^{1}, \ldots, f^{N}\right)^{T} \in C_{c}^{\infty}(\Omega)^{N}$, the function $\boldsymbol{u}=\left(u^{1}, \ldots, u^{N}\right)^{T}$ given by

$$
\boldsymbol{u}(x):=\int_{\Omega} \boldsymbol{G}(x, y) \boldsymbol{f}(y) d y \quad\left(i . e ., u^{i}(x):=\int_{\Omega} G_{i j}(x, y) f^{j}(y) d y\right)
$$

is a unique weak solution in $Y_{0}^{1,2}(\Omega)^{N}$ of $L \boldsymbol{u}=-\boldsymbol{f}$.

Moreover, $\boldsymbol{G}(x, y)$ satisfies the following pointwise estimate:

$$
|\boldsymbol{G}(x, y)| \leq C\left(\frac{1}{\gamma R^{2}}+\ln \frac{R}{|x-y|}\right) \quad \text { if }|x-y|<R:=\frac{1}{2} \max \left(d_{x}, d_{y}\right),
$$

where $d_{x}:=\operatorname{dist}(x, \partial \Omega)$ and $C=C(\lambda, \Lambda, N)<\infty$. Consequently, $\boldsymbol{G}(\cdot, y)$ and $\boldsymbol{G}(x, \cdot)$ belong to $L^{p}\left(B_{r}(y) \cap \Omega\right)$ and $L^{p}\left(B_{r}(x) \cap \Omega\right)$, respectively, for all $r>0$ and $p \in[1, \infty)$. Furthermore, $D \boldsymbol{G}(\cdot, y)$ and $\boldsymbol{D} \boldsymbol{G}(x, \cdot)$ belong to $L^{p}\left(B_{r}(y) \cap \Omega\right)$ and $L^{p}\left(B_{r}(x) \cap \Omega\right)$, respectively, for all $r>0$ and $p \in[1,2)$. Finally, we have the following symmetry relation:

$$
\left.\boldsymbol{G}(y, x)={ }^{t} \boldsymbol{G}(x, y)^{T} \quad \text { (i.e., } G_{i j}(y, x)={ }^{t} G_{j i}(x, y)\right),
$$

where ${ }^{t} \boldsymbol{G}(x, y)$ is the Green's matrix of the transpose operator ${ }^{t} L$ in $\Omega$. 
Remark 2.19. When $|\Omega|<\infty$, we have global $L^{p}$ estimates for the Green's matrix and its derivatives. In that case, it will be evident from the proof of Theorem 2.12 that $\boldsymbol{G}(\cdot, y)$ and $\boldsymbol{G}(x, \cdot)$ belong to $L^{p}(\Omega)$ for all $p \in[1, \infty)$ and that $D \boldsymbol{G}(\cdot, y)$ and $D \boldsymbol{G}(x, \cdot)$ belong to $L^{p}(\Omega)$ for all $p \in[1,2)$.

Next, we consider a domain above a Lipschitz graph. Let $\Omega$ be given by

$$
\Omega=\left\{\left(x_{1}, x_{2}\right) \in \mathbb{R}^{2}: x_{2}>\varphi\left(x_{1}\right)\right\},
$$

where $\varphi: \mathbb{R} \rightarrow \mathbb{R}$ is a Lipschitz function with a Lipschitz constant $M:=\left\|\varphi^{\prime}\right\|_{\infty}<$ $\infty$.

Theorem 2.21. Let the operator L satisfy the conditions (2.2) and (2.3). Assume that $\Omega$ is given by (2.20). Then, there exists a unique Green's matrix $\boldsymbol{G}(x, y)$ satisfying all the properties of Theorem 2.12 except (2.17). Instead of (2.17) from Theorem 2.12, we have

$$
|\boldsymbol{G}(x, y)| \leq C \min \left\{1+\ln _{+}\left(d_{x, y} /|x-y|\right), d_{x, y}^{\mu}|x-y|^{-\mu}\right\} \quad \forall x, y \in \Omega, \quad x \neq y,
$$

where $d_{x, y}:=\min \left(d_{x}, d_{y}\right), \quad d_{x}:=\operatorname{dist}(x, \partial \Omega), \ln _{+} t:=\max (\ln t, 0), C=$ $C(\lambda, \Lambda, N, M)<\infty$, and $\mu=\mu(\lambda, \Lambda, M) \in(0,1)$. In particular, (2.22) implies $\boldsymbol{G}(x, y) \rightarrow 0$ as $|x-y| \rightarrow \infty$.

\section{Proof of Theorem 2.12}

Throughout this section, we employ the letter $C$ to denote a constant depending on $\lambda, \Lambda, N$ while we use $C(\alpha, \beta, \ldots)$ to denote a constant depending on quantities $\alpha, \beta, \ldots$, as well as $\lambda, \Lambda, N$. It should be understood that $C$ may vary from line to line.

Let us recall the following version of Poincaré inequality (see, e.g., 8 for the proof).

Lemma 3.1. If $\Omega \subset \mathbb{R}^{2}$ is an open connected set with either finite volume or finite width, let $\gamma$ be given as in (2.13) of Theorem 2.12. Then

$$
\|u\|_{L^{2}(\Omega)} \leq(2 \gamma)^{-1 / 2}\|D u\|_{L^{2}(\Omega)} \quad \forall u \in W_{0}^{1,2}(\Omega) .
$$

By using the above lemma, one can show that if $\Omega$ has either finite volume or finite width, then $\Omega$ is a Green domain and $Y_{0}^{1,2}(\Omega)=W_{0}^{1,2}(\Omega)$ (see, e.g., [17, $\S 1.3 .4])$. In the rest of this section we shall identify $Y_{0}^{1,2}(\Omega)$ with $W_{0}^{1,2}(\Omega)$.

3.1. Construction of the Green's matrix. Let $\boldsymbol{\Gamma}(t, x, s, y) \quad(x, y \in \Omega$ and $t, s \in$ $\mathbb{R})$ be the parabolic Green's matrix given as in [3, Corollary 2.9]. Note that we have $\boldsymbol{\Gamma}(t, x, s, y)=\boldsymbol{\Gamma}(t-s, x, 0, y)$. Throughout the paper, we shall denote

$$
\begin{aligned}
\boldsymbol{K}(t, x, y) & :=\boldsymbol{\Gamma}(t, x, 0, y), \\
\tilde{\boldsymbol{K}}(t, x, y) & :=\int_{0}^{t} \boldsymbol{K}(s, x, y) d s .
\end{aligned}
$$


Here we record some properties of $\boldsymbol{K}(t, x, y)(x, y \in \Omega$ and $t \in \mathbb{R})$ derived in 3 , Corollary 2.9] for the reference. Recall that $d_{x}:=\operatorname{dist}(x, \partial \Omega)$ for $x \in \Omega$.

$$
\sup _{t \in\left(r^{2}, \infty\right)} \int_{\Omega}|\boldsymbol{K}(t, x, y)|^{2} d x \leq C r^{-2} \quad \forall y \in \Omega \quad \forall r<d_{y}
$$

$$
\iint_{(0, \infty) \times \Omega \backslash\left(0, r^{2}\right) \times B_{r}(y)}|\boldsymbol{K}(t, x, y)|^{4} d x d t \leq C r^{-4} \quad \forall y \in \Omega \quad \forall r<d_{y},
$$

$$
\iint_{(0, \infty) \times \Omega \backslash\left(0, r^{2}\right) \times B_{r}(y)}\left|D_{x} \boldsymbol{K}(t, x, y)\right|^{2} d x d t \leq C r^{-2} \quad \forall y \in \Omega \quad \forall r<d_{y},
$$

$$
\iint_{\left(0, r^{2}\right) \times B_{r}(y)}|\boldsymbol{K}(t, x, y)|^{p} d x d t \leq C(p) r^{-2 p+4} \quad \forall y \in \Omega \quad \forall r<d_{y} \quad \forall p \in[1,2),
$$

$$
\iint_{\left(0, r^{2}\right) \times B_{r}(y)}\left|D_{x} \boldsymbol{K}(t, x, y)\right|^{p} d x d t \leq C(p) r^{-3 p+4} \quad \forall y \in \Omega \quad \forall r<d_{y} \quad \forall p \in[1,4 / 3),
$$

$$
|\boldsymbol{K}(t, x, y)| \leq C\{\max (\sqrt{|t|},|x-y|)\}^{-2} \text { if } 0<\max (\sqrt{|t|},|x-y|)<\frac{1}{2} \max \left(d_{x}, d_{y}\right) .
$$

We define the Green's matrix $\boldsymbol{G}(x, y)$ as follows:

$$
\boldsymbol{G}(x, y):=\lim _{t \rightarrow \infty} \tilde{\boldsymbol{K}}(t, x, y)=\int_{0}^{\infty} \boldsymbol{K}(s, x, y) d s \quad \forall x, y \in \Omega, \quad x \neq y .
$$

The next lemma will show that $\boldsymbol{G}(x, y)$ is well defined.

Lemma 3.12. For any $x, y \in \Omega$ with $x \neq y$, we have $\int_{0}^{\infty}|\boldsymbol{K}(s, x, y)| d s<\infty$.

Proof. By [3, Theorem 2.7], we know that $t \mapsto \boldsymbol{K}(t, x, y)$ is continuous in $t \in \mathbb{R}$ for $x \neq y$. Therefore, we only need to show that $\int_{a}^{\infty}|\boldsymbol{K}(t, x, y)| d t<\infty$ for some $a>0$. Let $\boldsymbol{u}$ be the $k$-th column of $\boldsymbol{K}(\cdot, \cdot, y)$. Then, by the local boundedness estimate (see [13])

$$
|\boldsymbol{u}(t, x)| \leq C\left(\int_{t-\rho^{2}}^{t} \int_{B_{\rho}(x)}|\boldsymbol{u}(s, y)|^{2} d y d s\right)^{1 / 2} \quad \forall t>\rho^{2} \quad \forall \rho<d_{x} .
$$

By (3.2) of Lemma 3.1 $I(t):=\int_{\Omega}|\boldsymbol{u}(t, \cdot)|^{2}$ satisfies

$$
I^{\prime}(t)=-2 \int_{\Omega} A_{i j}^{\alpha \beta} D_{\beta} u^{j} D_{\alpha} u^{i}(t, \cdot) \leq-2 \lambda \int_{\Omega}|D \boldsymbol{u}(t, \cdot)|^{2} \leq-4 \lambda \gamma I(t) \quad \forall t>0 .
$$

Therefore, by using (3.5) we obtain

$$
\int_{\Omega}|\boldsymbol{K}(t, \cdot, y)|^{2} \leq C e^{-4 \lambda \gamma\left(t-r^{2}\right)} r^{-2} \quad \forall t>r^{2} \quad \forall r<d_{y} .
$$

By combining (3.13) and (3.14) we have

$$
|\boldsymbol{K}(t, x, y)| \leq A e^{-2 \lambda \gamma t} \quad \forall t>a,
$$

where $A=A\left(d_{x}, d_{y}\right)<\infty$ and $a=a\left(d_{x}, d_{y}\right)<\infty$. The lemma is proved. 
Next, we show that $\boldsymbol{G}(\cdot, y)$ is continuous in $\Omega \backslash\{y\}$ for any $y \in \Omega$. We need the following lemma, the proof of which can be found in [13, Theorem 3.3] (cf. (2.20) and (2.21) in [3]).

Lemma 3.16. Let $L$ satisfy (2.2) and (2.3). If $\boldsymbol{u}(t, x)$ is a weak solution of $\boldsymbol{u}_{t}-$ $L \boldsymbol{u}=0$ in $Q_{2 r}^{-}:=Q_{2 r}^{-}\left(\left(t_{0}, x_{0}\right)\right) \quad\left(:=\left(t_{0}-4 r^{2}, t_{0}\right) \times B_{2 r}\left(x_{0}\right)\right)$, then for all $x, x^{\prime} \in$ $B_{r}\left(x_{0}\right)$ and $t \in\left(t_{0}-r^{2}, t_{0}\right)$,

$$
\begin{aligned}
& \left|\boldsymbol{u}(x, t)-\boldsymbol{u}\left(x^{\prime}, t\right)\right| \leq C\left|x-x^{\prime}\right|^{\mu} r^{-(1+\mu)}\left\|D_{x} \boldsymbol{u}\right\|_{L^{2}\left(Q_{2 r}^{-}\right)}, \\
& \left|\boldsymbol{u}(x, t)-\boldsymbol{u}\left(x^{\prime}, t\right)\right| \leq C\left|x-x^{\prime}\right|^{\mu} r^{-(2+\mu)}\|\boldsymbol{u}\|_{L^{2}\left(Q_{2 r}^{-}\right)}
\end{aligned}
$$

where $\mu=\mu(\lambda, \Lambda) \in(0,1)$.

Let $\boldsymbol{u}(t, x)$ be the $k$-th column of $\boldsymbol{K}(t, x, y)$. Fix $x_{0} \in \Omega$ with $x_{0} \neq y$ and choose $r>0$ such that $r<d_{y}$ and $B_{2 r}\left(x_{0}\right) \subset \Omega \backslash B_{r}(y)$. By [3, Therem 2.7] and (3.3), we find that $\boldsymbol{u}(t, x)$ is a weak solution of $\boldsymbol{u}_{t}-L \boldsymbol{u}=0$ in $Q_{2 r}^{-}\left(\left(t_{0}, x_{0}\right)\right)$ for any $t_{0} \in \mathbb{R}$. Therefore, by using Lemma 3.16, (3.5), (3.7), and (3.14) we have (recall that $\boldsymbol{K}(t, x, y) \equiv 0$ for $t<0)$

$\left|\boldsymbol{K}(t, x, y)-\boldsymbol{K}\left(t, x_{0}, y\right)\right| \leq C\left|x-x_{0}\right|^{\mu} r^{-(2+\mu)} \quad \forall x \in B_{r}\left(x_{0}\right) \quad \forall t \in \mathbb{R}$,

$\left|\boldsymbol{K}(t, x, y)-\boldsymbol{K}\left(t, x_{0}, y\right)\right| \leq C\left|x-x_{0}\right|^{\mu} r^{-(2+\mu)} e^{-2 \lambda \gamma\left(t-r^{2}\right)} \quad \forall x \in B_{r}\left(x_{0}\right) \quad \forall t>5 r^{2}$.

Then, for any $x \in B_{r}\left(x_{0}\right)$, we have

$$
\begin{aligned}
\left|\boldsymbol{G}(x, y)-\boldsymbol{G}\left(x_{0}, y\right)\right| \leq & \int_{0}^{5 r^{2}}\left|\boldsymbol{K}(t, x, y)-\boldsymbol{K}\left(t, x_{0}, y\right)\right| d t \\
& +\int_{5 r^{2}}^{\infty}\left|\boldsymbol{K}(t, x, y)-\boldsymbol{K}\left(t, x_{0}, y\right)\right| d t \\
\leq & C\left|x-x_{0}\right|^{\mu} r^{-\mu}\left(1+r^{-2} \gamma^{-1}\right) .
\end{aligned}
$$

Therefore, we find that $\boldsymbol{G}(\cdot, y)$ is locally Hölder continuous in $\Omega \backslash\{y\}$. Let ${ }^{t} \boldsymbol{G}(x, y)$ be the Green's matrix of the transpose operator ${ }^{t} L$ in $\Omega$; i.e.,

$$
{ }^{t} \boldsymbol{G}(x, y):=\lim _{t \rightarrow \infty}{ }^{t} \tilde{\boldsymbol{K}}(t, x, y)=\int_{0}^{\infty}{ }^{t} \boldsymbol{K}(s, x, y) d s \quad \forall x, y \in \Omega, \quad x \neq y,
$$

where ${ }^{t} \boldsymbol{K}(t, x, y)$ and ${ }^{t} \tilde{\boldsymbol{K}}(t, x, y)$ are defined similarly as in (3.3) and (3.4). Let ${ }^{t} \boldsymbol{\Gamma}(s, y, t, x)$ be the parabolic Green's matrix of ${ }^{t} \mathcal{L}:=-\partial_{t}-{ }^{t} L$ constructed as in [3. Then by [3, Lemma 3.5]

$$
{ }^{t} \boldsymbol{K}(t, x, y)={ }^{t} \boldsymbol{\Gamma}(-t, x, 0, y)=\boldsymbol{\Gamma}(0, y,-t, x)^{T}=\boldsymbol{K}(t, y, x)^{T},
$$

and thus we conclude that

$$
{ }^{t} \tilde{\boldsymbol{K}}(t, x, y)=\tilde{\boldsymbol{K}}(t, y, x)^{T} \text { and }{ }^{t} \boldsymbol{G}(x, y)=\boldsymbol{G}(y, x)^{T} .
$$

In particular, we proved (2.18). Since ${ }^{t} L$ satisfies (2.2) and (2.3) with the same $\lambda, \Lambda$, we find as in (3.19) that ${ }^{t} \boldsymbol{G}(\cdot, x)$ is locally Hölder continuous in $\Omega \backslash\{x\}$ for all $x \in \Omega$. Therefore, by (2.18) we conclude that $\boldsymbol{G}(x, y)$ is continuous in $\{(x, y) \in \Omega \times \Omega: x \neq y\}$.

Next, we prove that $\boldsymbol{G}(x, \cdot)$ is locally integrable for all $x \in \Omega$ and that $\boldsymbol{u}$ defined by (2.16) is a weak solution in $W_{0}^{1,2}(\Omega)^{N}$ of $L \boldsymbol{u}=-\boldsymbol{f}$. 
Lemma 3.23. The following estimates hold uniformly for all $t>0$ and for all $y \in \Omega$ :

$$
\begin{aligned}
& \|\tilde{\boldsymbol{K}}(t, \cdot, y)\|_{L^{p}\left(B_{\rho}(y)\right)} \leq C(p) \gamma^{-1} \rho^{2 / p-2} \quad \forall p \in[1,2), \quad \text { where } \rho=d_{y} \\
& \|\tilde{\boldsymbol{K}}(t, \cdot, y)\|_{L^{4}\left(\Omega \backslash B_{r}(y)\right)} \leq C \gamma^{-1} r^{-3 / 2} \quad \forall r \leq d_{y}, \\
& \|D \tilde{\boldsymbol{K}}(t, \cdot, y)\|_{L^{p}\left(B_{\rho}(y)\right)} \leq C(p) \gamma^{-1} \rho^{2 / p-3} \quad \forall p \in[1,4 / 3), \quad \text { where } \rho=d_{y} \\
& \|D \tilde{\boldsymbol{K}}(t, \cdot, y)\|_{L^{2}\left(\Omega \backslash B_{r}(y)\right)} \leq C \gamma^{-1} r^{-2} \quad \forall r \leq d_{y} .
\end{aligned}
$$

Proof. We begin by proving (3.24). Fix $p \in[1,2)$. By Minkowski's inequality, we have

$$
\begin{aligned}
\left(\int_{B_{\rho}(y)}|\tilde{\boldsymbol{K}}(t, x, y)|^{p} d x\right)^{1 / p} & \leq \int_{0}^{t}\left(\int_{B_{\rho}(y)}|\boldsymbol{K}(s, x, y)|^{p} d x\right)^{1 / p} d s \\
& \leq \int_{0}^{\rho^{2}}+\int_{\rho^{2}}^{\infty}\left(\int_{B_{\rho}(y)}|\boldsymbol{K}(s, x, y)|^{p} d x\right)^{1 / p} d s:=I_{1}+I_{2} .
\end{aligned}
$$

We estimate $I_{1}$ by using Hölder's inequality and (3.8) as follows:

$$
I_{1} \leq\left(\int_{0}^{\rho^{2}} \int_{B_{\rho}(y)}|\boldsymbol{K}(s, x, y)|^{p} d x d s\right)^{1 / p} \rho^{2(1-1 / p)} \leq C(p) \rho^{2 / p} .
$$

To estimate $I_{2}$, observe that Hölder's inequality and (3.14) yield (recall $1 \leq p<2$ )

$$
\begin{aligned}
\left(\int_{B_{\rho}(y)}|\boldsymbol{K}(s, x, y)|^{p} d x\right)^{1 / p} & \leq\left(\int_{B_{\rho}(y)}|\boldsymbol{K}(s, x, y)|^{2} d x\right)^{1 / 2}\left|B_{\rho}(y)\right|^{1 / p-1 / 2} \\
& \leq C(p) \rho^{2 / p-2} e^{-2 \lambda \gamma\left(s-\rho^{2}\right)} \quad \forall s>\rho^{2} .
\end{aligned}
$$

Therefore, we obtain

$$
I_{2} \leq C(p) \rho^{2 / p-2} \int_{\rho^{2}}^{\infty} e^{-2 \lambda \gamma\left(s-\rho^{2}\right)} d s \leq C(p) \rho^{2 / p-2} \gamma^{-1} .
$$

Since $1 \leq \rho^{-2} \gamma^{-1}$ in any case, we obtain (3.24) by combining (3.29) and (3.30).

Next, we prove (3.25). By using Minkowski's inequality as in (3.28), we have

$$
\begin{gathered}
\left(\int_{\Omega \backslash B_{r}(y)}|\tilde{\boldsymbol{K}}(t, x, y)|^{4} d x\right){ }^{1 / 4} \leq \int_{0}^{r^{2}}+\int_{r^{2}}^{\infty}\left(\int_{\Omega \backslash B_{r}(y)}|\boldsymbol{K}(s, x, y)|^{4} d x\right)^{1 / 4} d s \\
:=I_{3}+I_{4} .
\end{gathered}
$$

By proceeding as in (3.29) but using (3.6) instead, we obtain

$$
I_{3} \leq\left(\int_{0}^{r^{2}} \int_{\Omega \backslash B_{r}(y)}|\boldsymbol{K}(s, x, y)|^{4} d x d s\right)^{1 / 4} r^{2(1-1 / 4)} \leq C r^{1 / 2} .
$$


By a well known embedding theorem (see, e.g., [14, §II.3] or [15, Theorem 6.9]), the energy inequality, and (3.14), we have for $t>r^{2}$

$$
\begin{aligned}
\int_{t}^{\infty} \int_{\Omega}|\boldsymbol{K}(s, x, y)|^{4} d x d s & \leq C\left(\sup _{t \leq s} \int_{\Omega}|\boldsymbol{K}(s, x, y)|^{2} d x\right) \int_{t}^{\infty} \int_{\Omega}\left|D_{x} \boldsymbol{K}(s, x, y)\right|^{2} d x d s \\
& \leq C\left(\int_{\Omega}|\boldsymbol{K}(t, x, y)|^{2} d x\right)^{2} \leq C r^{-4} e^{-8 \lambda \gamma\left(t-r^{2}\right)} .
\end{aligned}
$$

Then, by using Hölder's inequality and (3.32) we estimate

$$
\begin{aligned}
I_{4} & \leq \sum_{j=1}^{\infty}\left(\int_{j r^{2}}^{(j+1) r^{2}} \int_{\Omega}|\boldsymbol{K}(s, x, y)|^{4} d x d s\right)^{1 / 4} r^{3 / 2} \leq C r^{1 / 2} \sum_{j=1}^{\infty} e^{-2 \lambda \gamma(j-1) r^{2}} \\
& \leq C r^{1 / 2}\left(1+r^{-2} \gamma^{-1}\right) .
\end{aligned}
$$

By combining (3.31) and (3.33), we get (3.25).

We now turn to the proof of (3.26). Fix $p \in[1,4 / 3)$. As in (3.28), we have

$$
\begin{aligned}
& \left(\int_{B_{\rho}(y)}\left|D_{x} \tilde{\boldsymbol{K}}(t, x, y)\right|^{p} d x\right)^{1 / p} \\
& \quad \leq \int_{0}^{\rho^{2}}+\int_{\rho^{2}}^{\infty}\left(\int_{B_{\rho}(y)}\left|D_{x} \boldsymbol{K}(s, x, y)\right|^{p} d x\right)^{1 / p} d s:=I_{5}+I_{6} .
\end{aligned}
$$

By Hölder's inequality and (3.9), we find

$$
I_{5} \leq\left(\int_{0}^{\rho^{2}} \int_{B_{\rho}(y)}\left|D_{x} \boldsymbol{K}(s, x, y)\right|^{p} d x d s\right)^{1 / p} \rho^{2(1-1 / p)} \leq C(p) \rho^{-1+2 / p} .
$$

To estimate $I_{6}$, note that Hölder's inequality implies (recall $1 \leq p<4 / 3$ )

$$
\left(\int_{B_{\rho}(y)}\left|D_{x} \boldsymbol{K}(s, x, y)\right|^{p}\right)^{1 / p} \leq\left(\int_{B_{\rho}(y)}\left|D_{x} \boldsymbol{K}(s, x, y)\right|^{2}\right)^{1 / 2}\left|B_{\rho}(y)\right|^{1 / p-1 / 2} .
$$

As in (3.32), the energy inequality and (3.14) yield

$$
\int_{t}^{\infty} \int_{\Omega}\left|D_{x} \boldsymbol{K}(s, x, y)\right|^{2} d x d s \leq C \rho^{-2} e^{-4 \lambda \gamma\left(t-\rho^{2}\right)} \quad \forall t>\rho^{2} .
$$

Then, as in (3.33), we estimate $I_{6}$ by combining (3.35) and (3.36):

$$
\begin{aligned}
I_{6} & \leq C(p) \rho^{2 / p} \sum_{j=1}^{\infty}\left(\int_{j \rho^{2}}^{(j+1) \rho^{2}} \int_{\Omega}\left|D_{x} \boldsymbol{K}(s, x, y)\right|^{2} d x d s\right)^{1 / 2} \\
& \leq C(p) \rho^{2 / p-1}\left(1+\rho^{-2} \gamma^{-1}\right) .
\end{aligned}
$$

We obtain (3.26) by adding (3.34) and (3.37). 
Finally, we prove (3.27). By using Minkowski's inequality again, we have

$$
\begin{aligned}
& \left(\int_{\Omega \backslash B_{r}(y)}\left|D_{x} \tilde{\boldsymbol{K}}(t, x, y)\right|^{2} d x\right)^{1 / 2} \\
& \quad \leq \int_{0}^{r^{2}}+\int_{r^{2}}^{\infty}\left(\int_{\Omega \backslash B_{r}(y)}\left|D_{x} \boldsymbol{K}(s, x, y)\right|^{2} d x\right)^{1 / 2} d s:=I_{7}+I_{8} .
\end{aligned}
$$

We estimate $I_{7}$ by using Hölder's inequality and (3.7):

$$
I_{7} \leq\left(\int_{0}^{r^{2}} \int_{\Omega \backslash B_{r}(y)}\left|D_{x} \boldsymbol{K}(s, x, y)\right|^{2} d x d s\right)^{1 / 2} r \leq C .
$$

Also, by using (3.36) and proceeding as in (3.37), we obtain

$$
I_{8} \leq r \sum_{j=1}^{\infty}\left(\int_{j r^{2}}^{(j+1) r^{2}} \int_{\Omega}\left|D_{x} \boldsymbol{K}(s, x, y)\right|^{2} d x d s\right)^{1 / 2} \leq C\left(1+r^{-2} \gamma^{-1}\right) .
$$

Therefore, (3.27) follows from (3.38) and (3.39). The lemma is proved.

Fix $p_{0} \in(1,2)$ and $r \leq d_{y}$. By (3.11) and (3.24), there exists a sequence $\left\{t_{m}\right\}_{m=1}^{\infty}$ tending to infinity such that $\tilde{\boldsymbol{K}}\left(t_{m}, \cdot, y\right) \rightarrow \boldsymbol{G}(\cdot, y)$ weakly in $L^{p_{0}}\left(B_{r}(y)\right)$, and thus we have

$$
\|\boldsymbol{G}(\cdot, y)\|_{L^{p_{0}\left(B_{r}(y)\right)}} \leq C\left(p_{0}, \gamma, d_{y}, r\right)<\infty \quad \forall r \leq d_{y} .
$$

By a similar reasoning, (3.25) yields that

$$
\|\boldsymbol{G}(\cdot, y)\|_{L^{4}\left(\Omega \backslash B_{r}(y)\right)} \leq C \gamma^{-1} r^{-3 / 2} \quad \forall r \in\left(0, d_{y}\right] .
$$

The above inequalities together with (2.18) imply that $\boldsymbol{G}(x, \cdot)$ is locally integrable for any $x \in \Omega$. Therefore, the integral in (2.16) is absolutely convergent for any $\boldsymbol{f} \in C_{c}^{\infty}(\Omega)$, and thus $\boldsymbol{u}$ is well defined in (2.16). Moreover, (3.24) and (3.25) together with (3.22) imply

$$
\boldsymbol{v}(t, x):=\int_{\Omega} \tilde{\boldsymbol{K}}(t, x, y) \boldsymbol{f}(y) d y
$$

is well defined. By the dominated convergence theorem, we also find that

$$
\lim _{t \rightarrow \infty} \boldsymbol{v}(t, x)=\int_{\Omega} \boldsymbol{G}(x, y) \boldsymbol{f}(y) d y=\boldsymbol{u}(x) .
$$

Also, by the definition of $\tilde{\boldsymbol{K}}(t, x, y)$ in (3.4), it is easy to verify

$$
\boldsymbol{v}_{t}(t, x)=\int_{\Omega} \boldsymbol{K}(t, x, y) \boldsymbol{f}(y) d y \quad \forall t>0 .
$$

Then, as in the proof of Lemma 3.12, we have

$$
\left\|\boldsymbol{v}_{t}(t, \cdot)\right\|_{L^{2}(\Omega)}^{2} \leq C e^{-4 \lambda \gamma t}\|\boldsymbol{f}\|_{L^{2}(\Omega)}^{2} \quad \forall t>0 .
$$

We need the following lemma to show that $\boldsymbol{u}$ is a weak solution in $W_{0}^{1,2}(\Omega)^{N}$ of $L \boldsymbol{u}=-\boldsymbol{f}$. The readers are asked to consult [3] or 14 for the definition of $\stackrel{\circ}{V}_{2}^{1,0}((0, T) \times \Omega)$, etc. 
Lemma 3.44. For all $T>0$, the function $\boldsymbol{v}$ defined in (3.40) is the unique weak solution in $\stackrel{\circ}{V}_{2}^{1,0}((0, T) \times \Omega)^{N}$ of the problem

$$
\boldsymbol{v}_{t}-L \boldsymbol{v}=\boldsymbol{f}, \quad \boldsymbol{v}=0 \text { on }(0, T) \times \partial \Omega, \quad \boldsymbol{v}(0, \cdot)=0 .
$$

Proof. Let $\boldsymbol{w}$ be the weak solution in $\stackrel{\circ}{2}_{2}^{1,0}((0, T) \times \Omega)^{N}$ of the problem (3.45), the existence and uniqueness of which can be found in [14. We only need to show that $\boldsymbol{v} \equiv \boldsymbol{w}$ in $(0, T) \times \Omega$. Fix $t \in(0, T)$ and $x \in \Omega$. Let ${ }^{t} \boldsymbol{\Gamma}(s, y, t, x)$ be the parabolic Green's matrix of ${ }^{t} \mathcal{L}:=-\partial_{t}-{ }^{t} L$ constructed as in $[3$. Then, by proceeding similarly as in the proof of [3, Theorem 2.7], we obtain (cf. [3, Lemma 3.1])

$$
\begin{aligned}
w^{k}(t, x) & =\int_{0}^{T} \int_{\Omega}{ }^{t} \Gamma_{i k}(s, y, t, x) f^{i}(y) d y d s=\int_{0}^{T} \int_{\Omega} \Gamma_{k i}(t, x, s, y) f^{i}(y) d y d s \\
& =\int_{0}^{t} \int_{\Omega} K_{k i}(t-s, x, y) f^{i}(y) d y d s=\int_{0}^{t} \int_{\Omega} K_{k i}(s, x, y) f^{i}(y) d y d s=v^{k}(t, x),
\end{aligned}
$$

where we have used $\boldsymbol{K}(t, x, y) \equiv 0$ for $t<0$. The lemma is proved.

Note that (3.43) particularly implies $\boldsymbol{v}_{t}(t, \cdot) \in L^{2}(\Omega)^{N}$, and thus it is not hard to verify

$$
\int_{\Omega} v_{t}^{i}(t, \cdot) \phi^{i}+\int_{\Omega} A_{i j}^{\alpha \beta} D v^{j}(t, \cdot) D_{\alpha} \phi^{i}=\int_{\Omega} f^{i} \phi^{i} \quad \forall \phi \in W_{0}^{1,2}(\Omega)^{N} \quad \forall t>0 .
$$

Then, by setting $\phi=\boldsymbol{v}(t, \cdot)$ above, we find that for almost all $t>0$,

$$
\begin{aligned}
\lambda\|D \boldsymbol{v}(t, \cdot)\|_{L^{2}(\Omega)}^{2} & \leq\left(\|\boldsymbol{f}\|_{L^{2}(\Omega)}+\left\|\boldsymbol{v}_{t}(t, \cdot)\right\|_{L^{2}(\Omega)}\right)\|\boldsymbol{v}(t, \cdot)\|_{L^{2}(\Omega)} \\
& \leq C \gamma^{-1 / 2}\|\boldsymbol{f}\|_{L^{2}(\Omega)}\|D \boldsymbol{v}(t, \cdot)\|_{L^{2}(\Omega)},
\end{aligned}
$$

where we have used (3.43) and Lemma 3.1 Therefore, for almost all $t>0$,

$$
\|D \boldsymbol{v}(t, \cdot)\|_{L^{2}(\Omega)} \leq C \gamma^{-1 / 2}\|\boldsymbol{f}\|_{L^{2}(\Omega)} .
$$

Then, by the weak compactness and (3.41), we find that there exists an increasing sequence $\left\{t_{m}\right\}_{m=1}^{\infty}$ tending to infinity such that

$$
\lim _{m \rightarrow \infty} \int_{\Omega} A_{i j}^{\alpha \beta} D v^{j}\left(t_{m}, \cdot\right) D_{\alpha} \phi^{i}=\int_{\Omega} A_{i j}^{\alpha \beta} D u^{j} D_{\alpha} \phi^{i} \quad \forall \phi \in W_{0}^{1,2}(\Omega)^{N} .
$$

Therefore, it follows from (3.43), (3.46), and (3.48) that $\boldsymbol{u}$ defined in (2.16) is a weak solution in $W_{0}^{1,2}(\Omega)^{N}$ of $L \boldsymbol{u}=-\boldsymbol{f}$.

Now, we prove the uniqueness. Suppose that there exists another matrix valued function $\tilde{\boldsymbol{G}}(x, y)$ such that $\tilde{\boldsymbol{G}}(x, y)$ is continuous in $\{(x, y) \in \Omega \times \Omega: x \neq y\}, \tilde{\boldsymbol{G}}(x, \cdot)$ is locally integrable in $\Omega$ for all $x \in \Omega$, and for all $\boldsymbol{f} \in C_{c}^{\infty}(\Omega)^{N}$, the function $\tilde{\boldsymbol{u}}(x):=\int_{\Omega} \tilde{\boldsymbol{G}}(x, y) \boldsymbol{f}(y) d y$ is a weak solution of $L \tilde{\boldsymbol{u}}=-\boldsymbol{f}$ in $W_{0}^{1,2}(\Omega)^{N}$. Then, the difference $\boldsymbol{w}:=\boldsymbol{u}-\tilde{\boldsymbol{u}}$ is a weak solution of $L \boldsymbol{w}=0$ in $W_{0}^{1,2}(\Omega)^{N}$, and thus $\boldsymbol{w} \equiv 0$. Therefore, we have

$$
\int_{\Omega}(\boldsymbol{G}-\tilde{\boldsymbol{G}})(x, y) \boldsymbol{f}(y) d y=0 \quad \forall \boldsymbol{f} \in C_{c}^{\infty}(\Omega)^{N} .
$$

We conclude from (3.49) that $\boldsymbol{G}(x, \cdot) \equiv \tilde{\boldsymbol{G}}(x, \cdot)$ in $\Omega \backslash\{x\}$ for all $x \in \Omega$, and thus $\boldsymbol{G}(x, y)=\tilde{\boldsymbol{G}}(x, y)$ for all $x, y \in \Omega$ with $x \neq y$. We have proved the uniqueness. 
3.2. Proof of identities (2.14) and (2.15). Let us first prove (2.14). From (3.3), (3.4) and the construction of the parabolic Green's matrix $\boldsymbol{\Gamma}(t, x, s, y)$ in [3], it follows that (cf. (3.3) in [3])

$$
\int_{\Omega} K_{k i}(t, \cdot, y) \phi^{i}+\int_{\Omega} A_{i j}^{\alpha \beta} D_{\beta} \tilde{K}_{j k}(t, \cdot, y) D_{\alpha} \phi^{i}=\phi^{k}(y) \quad \forall \phi \in C_{c}^{\infty}(\Omega)^{N} .
$$

We note that (3.14) yields

$$
\lim _{t \rightarrow \infty} \int_{\Omega} K_{k i}(t, \cdot, y) \phi^{i}=0 \quad \forall k=1, \ldots, N .
$$

If we write $\phi=\zeta \phi+(1-\zeta) \phi$, where $\zeta \in C_{c}^{\infty}\left(B_{r}(y)\right)$ such that $\zeta \equiv 1$ on $B_{r / 2}(y)$, then

$$
\begin{aligned}
& \int_{\Omega} A_{i j}^{\alpha \beta} D_{\beta} \tilde{K}_{j k}(t, \cdot, y) D_{\alpha} \phi^{i}=\int_{B_{r}(y)} A_{i j}^{\alpha \beta} D_{\beta} \tilde{K}_{j k}(t, \cdot, y) D_{\alpha}\left(\zeta \phi^{i}\right) \\
& \quad+\int_{\Omega \backslash B_{r / 2}(y)} A_{i j}^{\alpha \beta} D_{\beta} \tilde{K}_{j k}(t, \cdot, y) D_{\alpha}\left((1-\zeta) \phi^{i}\right):=I_{1}(t)+I_{2}(t) .
\end{aligned}
$$

By Lemma 3.23 and (3.11), we find that there exists an increasing sequence $\left\{t_{m}\right\}_{m=1}^{\infty}$ tending to infinity such that

$$
\begin{aligned}
& \lim _{m \rightarrow \infty} I_{1}\left(t_{m}\right)=\int_{B_{r}(y)} A_{i j}^{\alpha \beta} D_{\beta} G_{j k}(\cdot, y) D_{\alpha}\left(\zeta \phi^{i}\right), \\
& \lim _{m \rightarrow \infty} I_{2}\left(t_{m}\right)=\int_{\Omega \backslash B_{r / 2}(y)} A_{i j}^{\alpha \beta} D_{\beta} G_{j k}(t, \cdot, y) D_{\alpha}\left((1-\zeta) \phi^{i}\right) .
\end{aligned}
$$

Therefore, by combining (3.50)-(3.54), we obtain (2.14).

Next, we prove (2.15). We claim

$$
\|(1-\eta) \tilde{\boldsymbol{K}}(t, \cdot, y)\|_{W^{1,2}(\Omega)} \leq C(\eta, \gamma)<\infty \quad \forall t>0,
$$

where $\eta \in C_{c}^{\infty}(\Omega)$ is such that $\eta \equiv 1$ on $B_{r}(y)$ for some $r<d_{y}$. Assume for the moment that the claim is true. Then, by the weak compactness and (3.11), there exists an increasing sequence $\left\{t_{m}\right\}_{m=1}^{\infty}$ tending to infinity such that

$$
(1-\eta) \tilde{\boldsymbol{K}}\left(t_{m}, \cdot, y\right) \rightarrow(1-\eta) \boldsymbol{G}(\cdot, y) \quad \text { weakly in } W^{1,2}(\Omega) .
$$

On the other hand, by [3. Theorem 2.7], we find that $(1-\eta) \tilde{\boldsymbol{K}}(t, \cdot, y) \in W_{0}^{1,2}(\Omega)$ for all $t>0$. Since $W_{0}^{1,2}(\Omega)$ is weakly closed in $W^{1,2}(\Omega)$, we have $(1-\eta) \boldsymbol{G}(\cdot, y) \in$ $W_{0}^{1,2}(\Omega)$, as desired. To complete the proof of (2.15), it remains to prove the claim (3.55). In fact, by Lemma 3.1, it is enough to show

$$
\|D((1-\eta) \tilde{\boldsymbol{K}}(t, \cdot, y))\|_{L^{2}(\Omega)} \leq C(\eta)<\infty \quad \forall t>0 .
$$

Let us prove (3.56). Assume that $\eta$ is supported in a ball $B \subset \mathbb{R}^{2}$. Then

$$
\begin{aligned}
\| D & ((1-\eta) \tilde{\boldsymbol{K}}(t, \cdot, y))\left\|_{L^{2}(\Omega)} \leq\right\| 1-\eta\left\|_{L^{\infty}}\right\| D \tilde{\boldsymbol{K}}(t, \cdot, y) \|_{L^{2}\left(\Omega \backslash B_{r}(y)\right)} \\
& +\|D \eta\|_{L^{\infty}}\|\tilde{\boldsymbol{K}}(t, \cdot, y)\|_{L^{2}\left(B \backslash B_{r}(y)\right)} \\
& \leq C(\eta)\|D \tilde{\boldsymbol{K}}(t, \cdot, y)\|_{L^{2}\left(\Omega \backslash B_{r}(y)\right)}+C(\eta)|B|^{1 / 4}\|\tilde{\boldsymbol{K}}(t, \cdot, y)\|_{L^{4}\left(\Omega \backslash B_{r}(y)\right)} \\
& \leq C(\eta) C(\gamma, r)=C(\eta, \gamma)<\infty \quad \forall t>0,
\end{aligned}
$$

where we have used Lemma 3.23 in the last step. This completes the proof of (3.56), and thus (2.15) is proved. 
3.3. Proof of logarithmic bound (2.17) and $L^{p}$ estimates. Without loss of generality, we may assume $d_{y} \geq d_{x}$; otherwise, in light of (2.18), we may exchange the role of $x$ and $y$. Note that if $|x-y|<R=d_{y} / 2$, then (3.10) yields

$$
|\boldsymbol{K}(t, x, y)| \leq C\{\max (\sqrt{t},|x-y|)\}^{-2} \quad \forall t \in\left(0, R^{2}\right) .
$$

On the other hand, if we set $\rho=R / 2$ in (3.13) and $r=\sqrt{3} R / 2$ in (3.14) (note that $\rho<d_{x}, r<d_{y}$, and $\rho^{2}+r^{2}=R^{2}$ ), then (3.15) becomes

$$
|\boldsymbol{K}(t, x, y)| \leq C R^{-2} e^{-2 \lambda \gamma\left(t-R^{2}\right)} \quad \forall t>R^{2} .
$$

Then, by using (3.58) and (3.59), we obtain (recall $|x-y|<R$ )

$$
\begin{aligned}
|\boldsymbol{G}(x, y)| & \leq C\left(\int_{0}^{|x-y|^{2}}|x-y|^{-2} d t+\int_{|x-y|^{2}}^{R^{2}} t^{-1} d t+\int_{R^{2}}^{\infty} R^{-2} e^{-2 \lambda \gamma\left(t-R^{2}\right)} d t\right) \\
& =C\left(1+2 \ln (R /|x-y|)+R^{-2}(2 \lambda \gamma)^{-1}\right) \\
& \leq C\left(R^{-2} \gamma^{-1}+\ln (R /|x-y|)\right) .
\end{aligned}
$$

We have thus proved (2.17). We now turn to the proof of local $p$-summability of $\boldsymbol{G}(\cdot, y)$ and $D \boldsymbol{G}(\cdot, y)$. Note that (3.60) particularly implies that

$$
\|\boldsymbol{G}(\cdot, y)\|_{L^{p}\left(B_{\rho}(y)\right)} \leq C\left(p, d_{y}, \gamma\right)<\infty \quad \forall \rho \in\left(0, d_{y} / 2\right] \quad \forall p \in[1, \infty) .
$$

We claim that $|D \boldsymbol{G}(\cdot, y)| \in L^{p}\left(B_{\rho}(y)\right)$ for all $0<\rho<d_{y}$ and $1 \leq p<2$. Let $\boldsymbol{u}$ be the $k$-th column of $\boldsymbol{G}(\cdot, y)$. Then, by (2.15), we have

$$
\boldsymbol{u} \in W^{1,2}\left(\Omega \backslash B_{\rho}(y)\right)^{N} \quad \forall \rho \in\left(0, d_{y}\right)
$$

and thus, by (2.14), we find that $\boldsymbol{u}$ is a weak solution of $L \boldsymbol{u}=0$ in $\Omega \backslash B_{\rho}(y)$ for any $\rho<d_{y}$. It follows from (2.17) that there is $r_{0}=r_{0}\left(\gamma, d_{y}\right)<1$ and $C_{0}=C_{0}\left(\gamma, d_{y}\right)<$ $\infty$ such that

$$
|\boldsymbol{G}(x, y)| \leq C_{0} \ln (1 /|x-y|) \quad \forall x \in B_{r_{0}}(y) .
$$

Fix $r<r_{0}$ and let $\zeta \in C_{c}^{\infty}\left(B_{r}(y)\right)$ be a cut-off function satisfying $\zeta \equiv 1$ on $\bar{B}_{r / 2}(y)$ and $|D \zeta| \leq C / r$. Then, by (2.15) we find

$$
(1-\zeta)^{2} \boldsymbol{u} \in W_{0}^{1,2}\left(\Omega^{\prime}\right)^{N}, \quad \text { where } \Omega^{\prime}:=\Omega \backslash \bar{B}_{r / 2}(y) .
$$

Since $\boldsymbol{u}$ is a weak solution in $W^{1,2}\left(\Omega^{\prime}\right)^{N}$ of $L \boldsymbol{u}=0$, by using (3.63) we have

$$
0=\int_{\Omega^{\prime}}(1-\zeta)^{2} A_{i j}^{\alpha \beta} D_{\beta} u^{j} D_{\alpha} u^{i}-\int_{\Omega^{\prime}} 2(1-\zeta) A_{i j}^{\alpha \beta} D_{\beta} u^{j} D_{\alpha} \zeta u^{i} .
$$

Therefore, by using the bound (3.62) we estimate

$$
\int_{\Omega \backslash B_{r}(y)}|D \boldsymbol{u}|^{2} \leq C r^{-2} \int_{B_{r}(y) \backslash B_{r / 2}(y)}|\boldsymbol{u}|^{2} \leq C C_{0}^{2}(\ln (r / 2))^{2} .
$$

Therefore, we have

$$
\int_{\Omega \backslash B_{r}(y)}|D \boldsymbol{G}(\cdot, y)|^{2} \leq C C_{0}^{2}(\ln (r / 2))^{2} \quad \forall r<r_{0} .
$$

Next, let $A_{t}=\left\{x \in \Omega:\left|D_{x} \boldsymbol{G}(x, y)\right|>t\right\}$ and choose $r=2 / t$. Then by (3.64)

$$
\left|A_{t} \backslash B_{r}(y)\right| \leq t^{-2} \int_{A_{t} \backslash B_{r}(y)}|D \boldsymbol{G}(\cdot, y)|^{2} \leq C C_{0}^{2} t^{-2}(\ln t)^{2} \quad \forall t>2 / r_{0}
$$


and $\left|A_{t} \cap B_{r}(y)\right| \leq\left|B_{r}(y)\right| \leq C t^{-2}$. Therefore, we conclude that for any $y \in \Omega$, there exist $C_{1}=C_{1}\left(\gamma, d_{y}\right)<\infty$ and $t_{0}=t_{0}\left(\gamma, d_{y}\right)>0$ such that

$$
\left|\left\{x \in \Omega:\left|D_{x} \boldsymbol{G}(x, y)\right|>t\right\}\right| \leq C_{1} t^{-2}(\ln t)^{2} \quad \forall t>t_{0} .
$$

From the estimates (3.65), it follows that $|D \boldsymbol{G}(\cdot, y)| \in L^{p}\left(B_{r}(y)\right)$ for all $r<d_{y}$ and for all $p \in[1,2)$ as we shall demonstrate below. Let $r<d_{y}$ be given and choose $\tau>t_{0}$. Note that

$$
\begin{aligned}
\int_{B_{r}(y)}|D \boldsymbol{G}(\cdot, y)|^{p}= & \int_{B_{r}(y) \cap\{|D \boldsymbol{G}(\cdot, y)| \leq \tau\}}|D \boldsymbol{G}(\cdot, y)|^{p} \\
& \quad+\int_{B_{r}(y) \cap\{|D \boldsymbol{G}(\cdot, y)|>\tau\}}|D \boldsymbol{G}(\cdot, y)|^{p} \\
\leq & \tau^{p}\left|B_{r}(y)\right|+\int_{\{|D \boldsymbol{G}(\cdot, y)|>\tau\}}|D \boldsymbol{G}(\cdot, y)|^{p} .
\end{aligned}
$$

By using (3.65), we estimate (recall $\tau>t_{0}$ )

$$
\begin{aligned}
\int_{\{|D \boldsymbol{G}(\cdot, y)|>\tau\}}|D \boldsymbol{G}(\cdot, y)|^{p} & =\int_{0}^{\infty} p t^{p-1}|\{|D \boldsymbol{G}(\cdot, y)|>\max (t, \tau)\}| d t \\
& \leq C_{1} \tau^{-2}(\ln \tau)^{2} \int_{0}^{\tau} p t^{p-1} d t+C_{1} \int_{\tau}^{\infty} p t^{p-3}(\ln t)^{2} d t
\end{aligned}
$$

Note that the above integrals converge if $0<p<2$, and thus we have shown that

$$
\int_{B_{r}(y)}|D \boldsymbol{G}(\cdot, y)|^{p} \leq C\left(p, \gamma, d_{y}, r\right)<\infty \quad \forall r \in\left(0, d_{y}\right) \quad \forall p \in[1,2) .
$$

On the other hand, (3.27) yields

$$
\|D \boldsymbol{G}(\cdot, y)\|_{L^{2}\left(\Omega \backslash B_{r}(y)\right)} \leq C \gamma^{-1} r^{-2} \quad \forall r \in\left(0, d_{y}\right) .
$$

By combining (3.66) and (3.67), we find

$$
\|D \boldsymbol{G}(\cdot, y)\|_{L^{p}\left(B_{r}(y) \cap \Omega\right)}<C\left(p, \gamma, d_{y}, r\right)<\infty \quad \forall r>0 \quad \forall p \in[1,2) .
$$

Next, for $r \geq d_{y} / 2$, fix a cut-off function $\zeta \in C_{c}^{\infty}\left(B_{2 r}(y) \backslash \bar{B}_{d_{y} / 4}(y)\right)$ such that $\zeta \equiv 1$ on $\bar{B}_{r}(y) \backslash B_{d_{y} / 2}(y)$ and $|D \zeta| \leq C / d_{y}$. By a similar computation as in (3.57), we have

$$
\|D(\zeta \boldsymbol{G}(\cdot, y))\|_{L^{2}\left(B_{2 r}(y) \cap \Omega\right)} \leq C\left(\gamma, d_{y}, r\right)<\infty .
$$

Since $\zeta \boldsymbol{G}(\cdot, y) \in W_{0}^{1,2}\left(B_{2 r}(y) \cap \Omega\right)$, the Sobolev inequality yields

$$
\|\boldsymbol{G}(\cdot, y)\|_{L^{p}\left(B_{r}(y) \cap \Omega \backslash \bar{B}_{d_{y} / 2}(y)\right)}<C\left(p, \gamma, d_{y}, r\right)<\infty \quad \forall p \in[1, \infty) .
$$

Then by combining (3.61) and (3.69), we obtain

$$
\|\boldsymbol{G}(\cdot, y)\|_{L^{p}\left(B_{r}(y) \cap \Omega\right)}<C\left(p, \gamma, d_{y}, r\right)<\infty \quad \forall r>0 \quad \forall p \in[1, \infty) .
$$


Finally, from (2.18), (3.68), and (3.70), it follows that $|D \boldsymbol{G}(x, \cdot)|$ belongs to $L^{p}\left(B_{r}(x) \cap \Omega\right)$ for all $r>0$ and $1 \leq p<2$ and that $|\boldsymbol{G}(x, \cdot)|$ belongs to $L^{p}\left(B_{r}(x) \cap \Omega\right)$ for all $r>0$ and $1 \leq p<\infty$. This completes the proof of the theorem.

\section{Proof of Theorem 2.21}

Throughout this section, we employ the letter $C$ to denote a constant depending on $\lambda, \Lambda, N$, and $M:=\left\|\varphi^{\prime}\right\|_{\infty}$. We use $C(\alpha, \beta, \ldots)$ to denote a constant depending on quantities $\alpha, \beta, \ldots$, as well as $\lambda, \Lambda, N, M$.

For $x=\left(x_{1}, x_{2}\right) \in \Omega$, where $\Omega$ is as in (2.20), we shall denote $\bar{x}:=\left(x_{1}, \varphi\left(x_{1}\right)\right) \in$ $\partial \Omega$. Note that $d_{x}$ is comparable to $|x-\bar{x}| ;$ more precisely, we have

$$
d_{x} \leq|x-\bar{x}| \leq \sqrt{1+M^{2}} d_{x} \quad \forall x \in \Omega .
$$

We shall use the following notation:

$$
\begin{aligned}
& P_{r}^{-}\left(t_{0}, x_{0}\right):=\left\{(t, x) \in \mathbb{R} \times \Omega: t_{0}-r^{2}<t<t_{0},\left|x-x_{0}\right|<r\right\}, \\
& S_{r}^{-}\left(t_{0}, x_{0}\right):=\mathbb{R} \times \partial \Omega \cap \partial P_{r}^{-}\left(t_{0}, x_{0}\right) .
\end{aligned}
$$

We ask the readers to consult [3] or [14] for the definition of the space $V_{2}$.

Lemma 4.4. Assume that the operator $L$ satisfies the conditions (2.2) and (2.3). Let $\Omega$ be given as in (2.20) and let $\bar{x}_{0} \in \partial \Omega$. Assume that $\boldsymbol{u}(t, x)$ is a weak solution in $V_{2}\left(P_{2 R}^{-}\left(t_{0}, \bar{x}_{0}\right)\right)$ of $\boldsymbol{u}_{t}-L \boldsymbol{u}=0$ and vanishes on $S_{2 R}^{-}\left(t_{0}, \bar{x}_{0}\right)$. Then, for any $y_{0} \in B_{R}\left(\bar{x}_{0}\right) \cap \Omega$, we have

$$
\int_{P_{\rho}^{-}\left(t_{0}, y_{0}\right)}\left|D_{x} \boldsymbol{u}\right|^{2} \leq C\left(\frac{\rho}{r}\right)^{2+2 \mu} \int_{P_{r}^{-}\left(t_{0}, y_{0}\right)}\left|D_{x} \boldsymbol{u}\right|^{2} \quad \forall \rho<r \leq R,
$$

where $\mu=\mu(\lambda, \Lambda, M) \in(0,1)$. As a consequence, for all $t \in\left(t_{0}-R^{2}, t_{0}\right)$, we have

$$
\left|\boldsymbol{u}(t, x)-\boldsymbol{u}\left(t, x^{\prime}\right)\right| \leq C\left|x-x^{\prime}\right|^{\mu} R^{-(1+\mu)}\left(\int_{P_{2 R}^{-}\left(t_{0}, \bar{x}_{0}\right)}\left|D_{x} \boldsymbol{u}\right|^{2}\right)^{1 / 2} \quad \forall x, x^{\prime} \in B_{R}\left(\bar{x}_{0}\right) .
$$

Proof. Let $\boldsymbol{v}(x)$ be a weak solution in $W^{1,2}\left(\Omega \cap B_{2 R}\left(\bar{x}_{0}\right)\right)$ of $L \boldsymbol{v}=0$ which vanishes on $\partial \Omega \cap B_{2 R}\left(\bar{x}_{0}\right)$. Let $y_{0} \in B_{R}\left(\bar{x}_{0}\right) \cap \Omega$. By a well-known boundary regularity theory for weak solutions of elliptic systems in two dimensional Lipschitz domains (see, e.g., [18]), we have

$$
\int_{B_{\rho}\left(y_{0}\right) \cap \Omega}|D \boldsymbol{v}|^{2} \leq C\left(\frac{\rho}{r}\right)^{2 \mu} \int_{B_{r}\left(y_{0}\right) \cap \Omega}|D \boldsymbol{v}|^{2} \quad \forall \rho<r \leq R,
$$

where $\mu=\mu(\lambda, \Lambda, M) \in(0,1)$. By a routine adjustment of an argument in 13, one can deduce (4.5) and (4.6) from (4.7).

Let $\Omega$ be given as in (2.20). It is rather tedious but routine to check that the estimate (4.5) allows us to treat $\Omega$ as if $\Omega=\mathbb{R}^{2}$ in the proof of [3, Theorem 2.7]. 
Consequently, we have the following estimates:

$$
|\boldsymbol{K}(t, x, y)| \leq C\left\{\max \left(|t|^{1 / 2},|x-y|\right)\right\}^{-2},
$$

$$
\int_{\Omega}|\boldsymbol{K}(t, x, y)|^{2} d x \leq C t^{-1} \quad \forall t>0
$$

$$
\iint_{(0, \infty) \times \Omega \backslash\left(0, r^{2}\right) \times B_{r}(y)}\left|D_{x} \boldsymbol{K}(t, x, y)\right|^{2} d x d t \leq C r^{-2} \quad \forall r>0,
$$

$$
\iint_{\left(0, r^{2}\right) \times\left(B_{r}(y) \cap \Omega\right)}\left|D_{x} \boldsymbol{K}(t, x, y)\right|^{p} d x d t \leq C(p) r^{-3 p+4} \quad \forall r>0 \quad \forall p \in[1,4 / 3) .
$$

To show the convergence of the integral in (3.11), we need the following lemma.

Lemma 4.12. Let $\Omega$ be given as in (2.20). There exists $\mu=\mu(\lambda, \Lambda, M) \in(0,1)$ such that

$$
|\boldsymbol{K}(t, x, y)| \leq C d_{x}^{\mu}\left\{\max \left(|t|^{1 / 2},|x-y|\right)\right\}^{-2-\mu} \quad \forall x, y \in \Omega, \quad x \neq y .
$$

Proof. Denote $r:=\max \left(|t|^{1 / 2},|x-y|\right)$. We may assume that $d_{x}<r /\left(10 \sqrt{1+M^{2}}\right)$; otherwise, (4.13) is an easy consequence of (4.8). Let $\boldsymbol{u}(t, x)$ be the $k$-th column of $\boldsymbol{K}(t, x, y)$ and set $R=r / 4$. Then by (4.1), (4.6), and (4.10), we have

$$
\begin{aligned}
& |\boldsymbol{u}(t, x)|=|\boldsymbol{u}(t, x)-\boldsymbol{u}(t, \bar{x})| \leq C|x-\bar{x}|^{\mu} R^{-1-\mu}\left(\iint_{(0, \infty) \times \Omega \backslash\left(0, R^{2}\right) \times B_{R}(y)}\left|D_{x} \boldsymbol{u}\right|^{2}\right)^{1 / 2} \\
& \quad \leq C|x-\bar{x}|^{\mu} R^{-2-\mu} \leq C d_{x}^{\mu} r^{-2-\mu} .
\end{aligned}
$$

We obtain (4.13) from (4.14). The lemma is proved.

Then, it follows from (4.13) that for all $x, y \in \Omega$ with $x \neq y$, we have

$$
\int_{0}^{\infty}|\boldsymbol{K}(t, x, y)| d t=\int_{0}^{|x-y|^{2}}+\int_{|x-y|^{2}}^{\infty}|\boldsymbol{K}(t, x, y)| d t \leq C d_{x}^{\mu}|x-y|^{-\mu}<\infty .
$$

Therefore, $\boldsymbol{G}(x, y)$, given the same as in (3.11), is well defined and satisfies

$$
|\boldsymbol{G}(x, y)| \leq C d_{x}^{\mu}|x-y|^{-\mu} \quad \forall x, y \in \Omega, \quad x \neq y .
$$

In fact, by using (4.8) and (4.13) together, we may obtain a better bound

$$
\begin{aligned}
|\boldsymbol{G}(x, y)| & \leq C\left(\int_{0}^{|x-y|^{2}}|x-y|^{-2} d t+\int_{|x-y|^{2}}^{d_{x}^{2}} t^{-1} d t+\int_{d_{x}^{2}}^{\infty} d_{x}^{\mu} t^{-1-\mu / 2} d t\right) \\
& \leq C\left(1+\ln \left(d_{x} /|x-y|\right)\right) \quad \text { if }|x-y|<d_{x} .
\end{aligned}
$$

Then by combining (4.16) and (4.17), we derive $\left(\right.$ recall $\left.\ln _{+} t:=\max (\ln t, 0)\right)$

$$
|\boldsymbol{G}(x, y)| \leq C \min \left\{1+\ln _{+}\left(d_{x} /|x-y|\right), d_{x}^{\mu}|x-y|^{-\mu}\right\} \quad \forall x, y \in \Omega, \quad x \neq y .
$$


Recall that (2.18) is a consequence of (3.21), which remains valid here. Therefore, (2.22) follows from (2.18) and (4.18). Note that (2.22) implies that for any $r>0$ and $p \in[1, \infty)$

$$
\|\boldsymbol{G}(x, \cdot)\|_{L^{p}\left(B_{r}(x) \cap \Omega\right)} \leq C\left(p, d_{x}, r\right)<\infty ; \quad\|\boldsymbol{G}(\cdot, y)\|_{L^{p}\left(B_{r}(y) \cap \Omega\right)} \leq C\left(p, d_{y}, r\right)<\infty .
$$

In particular, we have shown that $\boldsymbol{G}(x, \cdot)$ is locally integrable for all $x \in \Omega$. Next, we show that $\boldsymbol{G}(\cdot, y)$ is locally Hölder continuous in $\Omega \backslash\{y\}$. Fix $x_{0} \in \Omega$ with $x_{0} \neq y$ and choose $r>0$ such that $r<d_{y}$ and $B_{2 r}\left(x_{0}\right) \subset \Omega \backslash B_{r}(y)$. Similarly as in (4.14), Lemma 4.4 yields

$$
\left|\boldsymbol{K}(t, x, y)-\boldsymbol{K}\left(t, x_{0}, y\right)\right| \leq C\left|x-x_{0}\right|^{\mu} t^{-(1+\mu / 2)} \quad \forall x \in B_{r}\left(x_{0}\right) \quad \forall t>t_{0},
$$

where $t_{0}:=8\left(r+\sqrt{1+M^{2}} d_{x_{0}}\right)^{2}$. Notice that (3.17) still remains true here. Therefore, by using (4.20) instead of (3.18) and proceeding as in (3.19), we obtain

$$
\left|\boldsymbol{G}(x, y)-\boldsymbol{G}\left(x_{0}, y\right)\right| \leq C\left|x-x_{0}\right|^{\mu} \quad \forall x \in B_{r}\left(x_{0}\right) .
$$

Then, it follows from (2.18) and (4.21) that $\boldsymbol{G}(x, y)$ is continuous in $\{(x, y) \in \Omega \times \Omega: x \neq y\}$.

Now let us prove that $\boldsymbol{u}$ defined as in (2.16) is a unique weak solution in $Y_{0}^{1,2}(\Omega)^{N}$ of $L \boldsymbol{u}=-\boldsymbol{f}$. First observe that $\Omega$ is a Green domain. Let $\boldsymbol{v}(t, x)$ be defined the same as in (3.40). Then as in (3.41), we have $\lim _{t \rightarrow \infty} \boldsymbol{v}(t, x)=\boldsymbol{u}(x)$. Also, $\boldsymbol{v}_{t}(t, x)$ has the same representation as in (3.42). Then, by (4.9) and Minkowski's inequality, we have

$$
\left\|\boldsymbol{v}_{t}(t, \cdot)\right\|_{L^{2}(\Omega)} \leq C t^{-1 / 2}\|\boldsymbol{f}\|_{L^{1}(\Omega)} \quad \forall t>0
$$

and thus, by Lemma 3.44 , we estimate

$$
\|\boldsymbol{v}(t, \cdot)\|_{L^{2}(\Omega)} \leq C\|\boldsymbol{f}\|_{L^{1}(\Omega)} \int_{0}^{t} s^{-1 / 2} d s \leq C t^{1 / 2}\|\boldsymbol{f}\|_{L^{1}(\Omega)} \quad \forall t>0 .
$$

Assume that $\boldsymbol{f}$ is supported in a ball $B \subset \mathbb{R}^{2}$. Then by setting $\phi=\boldsymbol{v}(t, \cdot)$ in (3.46), we get

$$
\lambda\|D \boldsymbol{v}(t, \cdot)\|_{L^{2}(\Omega)}^{2} \leq\left\|\boldsymbol{v}_{t}(t, \cdot)\right\|_{L^{2}(\Omega)}\|\boldsymbol{v}(t, \cdot)\|_{L^{2}(\Omega)}+\|\boldsymbol{f}\|_{L^{2}(\Omega \cap B)}\|\boldsymbol{v}(t, \cdot)\|_{L^{2}(\Omega \cap B)}
$$

where we have used (4.22), (4.23), and (2.6). Then by applying Cauchy-Schwarz inequality to (4.24), we find

$$
\|D \boldsymbol{v}(t, \cdot)\|_{L^{2}(\Omega)}^{2} \leq C(\Omega, B)\left(\|\boldsymbol{f}\|_{L^{1}(\Omega \cap B)}^{2}+\|\boldsymbol{f}\|_{L^{2}(\Omega \cap B)}^{2}\right) \leq C(\Omega, B)\|\boldsymbol{f}\|_{L^{2}(\Omega)}^{2} \quad \forall t>0 .
$$

Therefore, by the weak compactness and (3.41), we conclude that there exists an increasing sequence $\left\{t_{m}\right\}_{m=1}^{\infty}$ tending to infinity such that $D \boldsymbol{v}\left(t_{m}, \cdot\right) \rightarrow D \boldsymbol{u}$ weakly in $L^{2}(\Omega)^{N}$ so that (3.48) holds. Also, by (4.25) we find $\|D \boldsymbol{u}\|_{L^{2}(\Omega)}<\infty$. By using (2.6) and (4.25) it is not hard to verify that for any $\zeta \in C_{c}^{\infty}\left(\mathbb{R}^{2}\right)$,

$$
\|\zeta \boldsymbol{v}(t, \cdot)\|_{W^{1,2}(\Omega)} \leq C(\zeta, \Omega, B)\|\boldsymbol{f}\|_{L^{2}(\Omega)}<\infty \quad \forall t>0 .
$$

Therefore, we conclude that $\zeta \boldsymbol{u} \in W_{0}^{1,2}(\Omega)^{N}$ for all $\zeta \in C_{c}^{\infty}(\Omega)$, and thus $\boldsymbol{u} \in$ $Y_{0}^{1,2}(\Omega)^{N}$. Consequently, it follows from (3.46), (3.48), (4.22), and Lemma 2.9 that $\boldsymbol{u}$ is a unique weak solution in $Y_{0}^{1,2}(\Omega)^{N}$ of $L \boldsymbol{u}=-\boldsymbol{f}$. 
By arguing the same as in the proof of Theorem 2.12, we get the uniqueness of the Green's matrix in $\Omega$.

We need the following lemma to prove (2.14) and (2.15).

Lemma 4.26. Let $\Omega$ be given as in (2.20). Then, for all $y \in \Omega$ and for all $t>0$, we have

$$
\begin{aligned}
& \|D \tilde{\boldsymbol{K}}(t, \cdot, y)\|_{L^{p}\left(B_{\rho}(y) \cap \Omega\right)} \leq C(p) \rho^{2 / p-1} \quad \forall \rho>0 \quad \forall p \in[1,4 / 3), \\
& \|D \tilde{\boldsymbol{K}}(t, \cdot, y)\|_{L^{2}\left(\Omega \backslash B_{r}(y)\right)} \leq C\left(1+d_{y}^{\mu} r^{-\mu}\right) \quad \forall r>0 .
\end{aligned}
$$

Proof. We proceed similarly as in the proof of Lemma 3.23. Let us begin by proving (4.27) first. By Minkowski's inequality, we have

$$
\begin{aligned}
& \left(\int_{B_{\rho}(y) \cap \Omega}\left|D_{x} \tilde{\boldsymbol{K}}(t, x, y)\right|^{p} d x\right)^{1 / p} \\
& \quad \leq \int_{0}^{\rho^{2}}+\int_{\rho^{2}}^{\infty}\left(\int_{B_{\rho}(y) \cap \Omega}\left|D_{x} \boldsymbol{K}(s, x, y)\right|^{p} d x\right)^{1 / p} d s:=I_{1}+I_{2} .
\end{aligned}
$$

Then, by Hölder's inequality and (4.11), we have

$$
I_{1} \leq\left(\int_{0}^{\rho^{2}} \int_{B_{\rho}(y) \cap \Omega}\left|D_{x} \boldsymbol{K}(s, x, y)\right|^{p} d x d s\right)^{1 / p} \rho^{2(1-1 / p)} \leq C(p) \rho^{-1+2 / p} .
$$

On the other hand, by using Hölder's inequality

$$
I_{2} \leq C(p) \rho^{2 / p} \sum_{j=1}^{\infty}\left(\int_{j \rho^{2}}^{(j+1) \rho^{2}} \int_{B_{\rho}(y) \cap \Omega}\left|D_{x} \boldsymbol{K}(s, x, y)\right|^{2} d x d s\right)^{1 / 2}:=C(p) \rho^{2 / p} \sum_{j=1}^{\infty} I_{2, j} .
$$

By setting $r=\sqrt{(j+1) / 2} \rho$ in (4.5) and using (4.10), we estimate

$$
\begin{aligned}
\left(I_{2, j}\right)^{2} & =\int_{P_{\rho}^{-}\left(2 r^{2}, y\right)}\left|D_{x} \boldsymbol{K}(t, x, y)\right|^{2} d x d t \\
& \leq C\left(\frac{\rho}{r}\right)^{2+2 \mu} \int_{P_{r}^{-}\left(2 r^{2}, y\right)}\left|D_{x} \boldsymbol{K}(t, x, y)\right|^{2} d x d t \\
& \leq C\left(\frac{\rho}{r}\right)^{2+2 \mu} \int_{(0, \infty) \times \Omega \backslash\left(0, r^{2}\right) \times B_{r}(y)}\left|D_{x} \boldsymbol{K}(t, x, y)\right|^{2} d x d t \\
& \leq C \rho^{2+2 \mu} r^{-4-2 \mu}=C \rho^{-2}(j+1)^{-2-\mu} .
\end{aligned}
$$

Therefore, by combining (4.30) and (4.31), we find

$$
I_{2} \leq C(p) \rho^{2 / p-1} \sum_{j=1}^{\infty}(j+1)^{-1-\mu / 2}=C(p) \rho^{2 / p-1},
$$

and thus (4.27) follows from (4.29) and (4.32).

Next, we turn to the proof of (4.28). As before, Minkowski's inequality yields

$$
\begin{aligned}
\left(\int_{\Omega \backslash B_{r}(y)}\left|D_{x} \tilde{\boldsymbol{K}}(t, x, y)\right|^{2} d x\right)^{1 / 2} & \leq \int_{0}^{r^{2}}+\int_{r^{2}}^{\infty}\left(\int_{\Omega \backslash B_{r}(y)}\left|D_{x} \boldsymbol{K}(s, x, y)\right|^{2} d x\right)^{1 / 2} d s \\
& :=I_{3}+I_{4} .
\end{aligned}
$$


Then, by Hölder's inequality and (4.10), we have

$$
I_{3} \leq\left(\int_{0}^{r^{2}} \int_{\Omega \backslash B_{r}(y)}\left|D_{x} \boldsymbol{K}(s, x, y)\right|^{2} d x d s\right)^{1 / 2} r \leq C .
$$

We need the following inequality to estimate $I_{4}$ :

$$
I_{5}(t):=\int_{t}^{\infty} \int_{\Omega}\left|D_{x} \boldsymbol{K}(s, x, y)\right|^{2} d x d s \leq C d_{y}^{2 \mu} t^{-1-\mu} \quad \forall r>0 \quad \forall t>0 .
$$

Let us momentarily assume that (4.34) holds and proceed similarly as in (4.31) to get

$$
\begin{aligned}
I_{4} & \leq\left(\int_{r^{2}}^{\infty} \int_{\Omega \backslash B_{r}(y)}\left|D_{x} \boldsymbol{K}(s, x, y)\right|^{2} d x d s\right)^{1 / 2} \\
& \leq \sum_{j=0}^{\infty} 2^{j / 2} r\left(\int_{2^{j} r^{2}}^{2^{j+1} r^{2}} \int_{\Omega}\left|D_{x} \boldsymbol{K}(s, x, y)\right|^{2} d x d s\right)^{1 / 2} \\
& \leq C d_{y}^{\mu} r^{-\mu} \sum_{j=0}^{\infty} 2^{-j \mu / 2} \leq C d_{y}^{\mu} r^{-\mu} .
\end{aligned}
$$

By combining (4.33) and (4.35), we obtain (4.28). It only remains to prove (4.34). Note that by (4.13) and (3.21) we have

$$
|\boldsymbol{K}(s, x, y)| \leq C d_{y}^{\mu}\left\{\max \left(s^{1 / 2},|x-y|\right)\right\}^{-2-\mu} \quad \forall x \neq y \quad \forall s>0 .
$$

Let $\zeta \in C^{\infty}(\mathbb{R})$ be such that $0 \leq \zeta \leq 1, \zeta \equiv 1$ on $[t, \infty), \zeta \equiv 0$ on $(-\infty, t / 2]$, and $\left|\zeta^{\prime}\right| \leq 4 / t$. Then, by the energy inequality (see, e.g., [14, §III.2]) and (4.36), we have

$$
\begin{aligned}
I_{5}(t) & \leq \int_{0}^{\infty} \int_{\Omega} \zeta(s)\left|D_{x} \boldsymbol{K}(s, x, y)\right|^{2} d y d s \leq C \int_{0}^{\infty} \int_{\Omega}\left|\zeta^{\prime}(s)\right||\boldsymbol{K}(s, x, y)|^{2} d y d s \\
& \leq C t^{-1} d_{y}^{2 \mu} \int_{t / 2}^{t}\left(\int_{|x-y|<\sqrt{s}} s^{-2-\mu} d y+\int_{|x-y| \geq \sqrt{s}}|x-y|^{-4-2 \mu} d y\right) d s \\
& \leq C t^{-1} d_{y}^{2 \mu} \int_{t / 2}^{t} s^{-1-\mu} d s \leq C d_{y}^{2 \mu} t^{-1-\mu} .
\end{aligned}
$$

This completes the proof of the lemma.

We now prove (2.14) and (2.15). To prove (2.14), first recall that (3.50) holds. By (4.9), we find that (3.51) remains valid. Assume that $\phi \in C_{c}^{\infty}(\Omega)^{N}$ is supported in $B_{R}(y) \cap \Omega$. By (3.11) and (4.27), we find that there is a sequence $\left\{t_{m}\right\}_{m=1}^{\infty}$ tending to infinity such that

$$
D \tilde{\boldsymbol{K}}\left(t_{m}, \cdot, y\right) \rightarrow D \boldsymbol{G}(\cdot, y) \quad \text { weakly in } L^{p}\left(B_{R}(y) \cap \Omega\right)^{N \times N} \text { for some } p>1 .
$$

Therefore, we find

$$
\lim _{m \rightarrow \infty} \int_{\Omega} A_{i j}^{\alpha \beta} D_{\beta} \tilde{K}_{j k}(\cdot, y) D_{\alpha} \phi^{i}=\int_{\Omega} A_{i j}^{\alpha \beta} D_{\beta} G_{j k}(\cdot, y) D_{\alpha} \phi^{i} .
$$

By combining (3.50), (3.51), and (4.37), we obtain (2.14). To prove (2.15), first observe that (4.28) yields

$$
\|D \boldsymbol{G}(\cdot, y)\|_{L^{2}\left(\Omega \backslash B_{r}(y)\right)} \leq C\left(d_{y}, r\right)<\infty \quad \forall r>0 .
$$


By using (4.19) and (4.38) and proceeding similarly as in (3.57), we obtain

$$
\|D((1-\eta) \boldsymbol{G}(\cdot, y))\|_{L^{2}(\Omega)} \leq C\left(\eta, d_{y}\right)<\infty .
$$

It follows from [3, Theorem 2.7] that for any $\zeta \in C_{c}^{\infty}\left(\mathbb{R}^{2}\right)$, we have

$$
\boldsymbol{F}(t, \cdot):=\zeta(1-\eta) \tilde{\boldsymbol{K}}(t, \cdot, y) \in W_{0}^{1,2}(\Omega)^{N \times N} \quad \forall t>0 .
$$

Clearly, $\lim _{t \rightarrow \infty} \boldsymbol{F}(t, \cdot)=\zeta(1-\eta) \boldsymbol{G}(\cdot, y)$. Moreover, by utilizing (3.22), (4.13), and (4.28), it is not hard to verify $\|\boldsymbol{F}(t, \cdot)\|_{W^{1,2}(\Omega)} \leq C\left(\zeta, \eta, d_{y}\right)<\infty$ for all $t>0$. Then, by a similar argument as in Section 3.2, we get $\zeta(1-\eta) \boldsymbol{G}(\cdot, y) \in W_{0}^{1,2}(\Omega)^{N \times N}$. We have proved (2.15).

Finally, notice that with (2.15) at hand, we may proceed similarly as in Section 3.3 to conclude that that $D \boldsymbol{G}(\cdot, y)$ and $D \boldsymbol{G}(x, \cdot)$ belong to $L^{p}\left(B_{r}(y) \cap \Omega\right)$ and $L^{p}\left(B_{r}(x) \cap \Omega\right)$, respectively, for all $r>0$ and $p \in[1,2)$. We have already seen in (4.19) that that $\boldsymbol{G}(\cdot, y)$ and $\boldsymbol{G}(x, \cdot)$ belong to $L^{p}\left(B_{r}(y) \cap \Omega\right)$ and $L^{p}\left(B_{r}(x) \cap \Omega\right)$, respectively, for all $r>0$ and $p \in[1, \infty)$. This completes the proof of the theorem.

\section{REMARK ON FUNDAMENTAL MATRICES}

In this section, we introduce a result of Auscher et al. 1] regarding construction of a fundamental matrix in $\mathbb{R}^{2}$. Let $\mathcal{H}^{1}\left(\mathbb{R}^{2}\right)$ be the usual Hardy space in $\mathbb{R}^{2}$ and $C_{0}\left(\mathbb{R}^{2}\right)$ be the space of continuous functions on $\mathbb{R}^{2}$ vanishing at infinity. For $x, y \in$ $\mathbb{R}^{2}, x \neq y$, define

$$
\boldsymbol{\Gamma}(x, y):=\int_{0}^{1} \boldsymbol{K}(t, x, y) d t+\int_{1}^{\infty}(\boldsymbol{K}(t, x, y)-\boldsymbol{K}(t, x, x)) d t .
$$

The following theorem appears in [1] as Theorem 3.16, where $L$ is assumed to be an elliptic operator with complex coefficients. With appropriate changes, the same proof carries over.

Theorem 5.2 (Auscher-McIntosh-Tchamitchian). Let the operator L satisfy (2.2) and (2.3). Then for all $x \in \mathbb{R}^{2}, \boldsymbol{\Gamma}(x, \cdot) \in B M O$, and for $\boldsymbol{f}=\left(f^{1}, \ldots, f^{N}\right)^{T} \in$ $\mathcal{H}^{1}\left(\mathbb{R}^{2}\right)^{N}$, the function defined by

$$
T \boldsymbol{f}(x):=\int_{\mathbb{R}^{2}} \boldsymbol{\Gamma}(x, y) \boldsymbol{f}(y) d y
$$

belongs to $C_{0}\left(\mathbb{R}^{2}\right)^{N}$. The linear map thus defined is continuous from $\mathcal{H}^{1}\left(\mathbb{R}^{2}\right)^{N}$ into $C_{0}\left(\mathbb{R}^{2}\right)^{N}$. Moreover, for all $\boldsymbol{f} \in \mathcal{H}^{1}\left(\mathbb{R}^{2}\right)^{N}, \boldsymbol{u}(x):=T \boldsymbol{f}(x)$ satisfies $\|D \boldsymbol{u}\|_{L^{2}\left(\mathbb{R}^{2}\right)}<$ $\infty$ and is a weak solution of $L \boldsymbol{u}=-\boldsymbol{f}$ in the sense of (2.10).

\section{ACKNOWLEDGMENTS}

The authors thank Steve Hofmann for very useful discussions.

\section{REFERENCES}

[1] Auscher, P.; McIntosh, A.; Tchamitchian, Ph. Heat kernels of second order complex elliptic operators and applications. J. Funct. Anal. 152 (1998), no. 1, 22-73. MR 1600066 (99e:47062)

[2] Chanillo, S.; Li, Y. Y. Continuity of solutions of uniformly elliptic equations in $R^{2}$. Manuscripta Math. 77 (1992), no. 4, 415-433. MR1190215 (93k:35033)

[3] Cho, S.; Dong, H.; Kim, S. On the Green's matrices of strongly parabolic systems of second order. arXiv:0705.1855; to appear in Indiana Univ. Math. J.

[4] Dolzmann, G.; Müller, S. Estimates for Green's matrices of elliptic systems by $L^{p}$ theory. Manuscripta Math. 88 (1995), no. 2, 261-273. MR1354111 (96g:35054) 
[5] Doob, J. L. Classical potential theory and its probabilistic counterpart. Reprint of the 1984 edition. Springer-Verlag, Berlin, 2001. MR 1814344 (2001j:31002)

[6] Escauriaza, L. Bounds for the fundamental solution of elliptic and parabolic equations in nondivergence form. Comm. Partial Differential Equations 25 (2000), no. 5-6, 821-845. MR.1759794 (2001i:35009)

[7] Fuchs, M. The Green matrix for strongly elliptic systems of second order with continuous coefficients. Z. Anal. Anwendungen 5 (1986), no. 6, 507-531. MR894243 (89a:35069)

[8] Gilbarg, D.; Trudinger, N. S. Elliptic partial differential equations of second order, Reprint of the 1998 ed. Springer-Verlag, Berlin, 2001. MR.1814364 (2001k:35004)

[9] Grüter, M.; Widman, K.-O. The Green function for uniformly elliptic equations. Manuscripta Math. 37 (1982), no. 3, 303-342. MR657523 (83h:35033)

[10] Hofmann, S.; Kim, S. The Green function estimates for strongly elliptic systems of second order. arXiv:0704.1352; to appear in Manuscripta Math. MR2341783

[11] Hofmann, S.; Kim, S. Gaussian estimates for fundamental solutions to certain parabolic systems. Publ. Mat. 48 (2004), no. 2, 481-496. MR2091016 (2005g:35004)

[12] Kenig, C.; Ni, W.-M. On the elliptic equation $L u-k+K \exp [2 u]=0$. Ann. Scuola Norm. Sup. Pisa Cl. Sci. (4) 12 (1985), no. 2, 191-224. MR829052 (87f:35065)

[13] Kim, S. Gaussian estimates for fundamental solutions of second order parabolic systems with time-independent coefficients. Trans. Amer. Math. Soc. 360 (2008), no. 11, 6031-6043. MR.2425701

[14] Ladyženskaja, O. A.; Solonnikov, V. A.; Ural'ceva, N. N. Linear and quasilinear equations of parabolic type. American Mathematical Society, Providence, RI, 1967. MR0241822 (39:3159b)

[15] Lieberman G. M. Second order parabolic differential equations, World Scientific Publishing Co., Inc., River Edge, NJ, 1996. MR1465184 (98k:35003)

[16] Littman, W.; Stampacchia, G.; Weinberger, H. F. Regular points for elliptic equations with discontinuous coefficients. Ann. Scuola Norm. Sup. Pisa (3) 17 (1963) 43-77. MR0161019 $(28: 4228)$

[17] Malý, J.; Ziemer, W. P. Fine regularity of solutions of elliptic partial differential equations. American Mathematical Society, Providence, RI, 1997. MR1461542 (98h:35080)

[18] Morrey, C. B., Jr. Multiple integrals in the calculus of variations. Springer-Verlag, New York, Inc., New York, 1966. MR0202511 (34:2380)

Division of Applied Mathematics, Brown University, 182 George Street, Providence, RHODE ISLAND 02912

E-mail address: hdong@brown.edu

Department of Mathematics, Yonsei University, 262 Seongsanno, Seodaemun-gu, SEOUL 120-749, Korea

E-mail address: kimseick@yonsei.ac.kr 